\title{
Correlation of fine needle aspiration cytology and frozen section biopsies in the diagnosis of thyroid nodules
}

\author{
Hung-Yu Chang, Jen-Der Lin, Jung-Fu Chen, Bie-Yu Huang, Chuen Hsueh,
} Long-Bin Jeng, Jir-Shiong Tsai

\begin{abstract}
Aims-To evaluate the correlation of fine needle aspiration (FNA) cytology and frozen section biopsy in the diagnosis of thyroid nodules.
\end{abstract}

Methods-The medical records of 662 patients who underwent FNA cytology of the thyroid and thyroid surgery were analysed. Frozen section biopsies were taken from 586 of the 662 patients. The diagnostic correlations of FNA cytology, frozen section, and both FNA cytology and frozen section with definitive histological assessment were evaluated.

Results-Among the 662 patients who received FNA cytology, there were 356 cases $(53.8 \%)$ diagnosed as benign, 114 cases $(17.2 \%)$ as malignant, 148 cases $(22.4 \%)$ as indeterminate, and 44 cases $(6.6 \%)$ as unsatisfactory. The positive predictive value for the detection of malignancy by FNA cytology was $92.1 \%$ and the negative predictive value was $95.2 \%$. The incidence of malignancy in the indeterminate cytological diagnosis was $23 \%$. The diagnosis from frozen sections was benign in 445 cases $(75.9 \%)$, malignant in 134 cases $(22.9 \%)$, and deferred in 7 cases $(1.2 \%)$. By frozen section, the positive and negative predictive values were $97 \%$ and $95.5 \%$, respectively. Diagnostic accuracy up to $98 \%$ was achieved when FNA cytology and frozen section diagnoses were in agreement. No false positives were observed when FNA cytology and frozen sections were both positive for malignancy. When FNA cytology and frozen section diagnoses were discordant, frozen section showed a higher accuracy $(78.9 \%)$ than FNA cytology $(21.1 \%)$. In the face of an indeterminate or unsatisfactory cytological diagnosis, the diagnostic accuracy of frozen sections reached $92.6 \%$.

Conclusions-The results confirm that FNA cytology is a useful tool in the initial evaluation of thyroid nodules. Intraoperative frozen section is a valuable procedure to confirm the cytological diagnosis and identify malignancy in patients with indeterminate or unsatisfactory cytological diagnosis. With reliance on frozen sections as an intraoperative guide of thyroid surgery, the possibility of unnecessary extensive surgery and the need for the second operation are considerably lower. (F Clin Pathol 1997;50:1005-1009)

Keywords: fine needle aspiration cytology; frozen section biopsy; thyroid nodule

Nodular thyroid disease is a common clinical problem in Taiwan. In a recent study, the adult goiter prevalence in Taiwan was $19.4 \%$ in men, $31.6 \%$ in women, and $25 \%$ in all people. ${ }^{1}$ It is estimated that approximately $5 \%$ to $10 \%$ of thyroid nodules are malignant, ${ }^{2}$ therefore the ability to distinguish benign from malignant nodules is vital to avoid unnecessary operations. Fine needle aspiration diagnosis aids the selection of patients for surgery and has become an important examination technique for the initial evaluation of thyroid nodules. ${ }^{3-14}$ Many surgeons use intraoperative frozen section biopsies to confirm the diagnosis of FNA cytology and guide the extent of thyroidectomy. ${ }^{3468}$ However, both FNA cytology and frozen sections are associated to some extent with false positive and false negative diagnoses. $^{5-8}$ To evaluate the contribution of both techniques to the diagnosis and management of benign and malignant thyroid nodules, we retrospectively reviewed a series of 662 patients who underwent both FNA cytology and thyroid surgery.

Patients and methods

Data were evaluated from 662 patients who underwent FNA cytology and surgical treatment for thyroid nodules at Chang Gung Memorial Hospital between January 1992 and August 1994. These patients were initially evaluated by endocrinologists or thyroid surgeons. Fine needle aspirations using 22 or 25 gauge disposable needles without local anaesthesia were performed by staff endocrinologists who prepared the smears from the aspirates. The smears were air dried and stained by modified Romanowsky method. ${ }^{15}$ Smears showing a minimum of six clusters of epithelial cells with more than 20 cells in each cluster were considered adequate. The cytological diagnoses were grouped into four categories: benign, malignant, indeterminate, and unsatisfactory. The term indeterminate was used synonomously with follicular neoplasm. Patients with a malignant or indeterminate cytological result were recommended to undergo surgery, whereas those with a benign cytological 
Table 1 Correlation of fine needle aspiration diagnoses with definitive histological diagnoses

\begin{tabular}{|c|c|c|c|c|c|c|c|}
\hline \multirow[b]{3}{*}{$\begin{array}{l}\text { Fine needle aspiration } \\
(n=662)\end{array}$} & \multicolumn{7}{|c|}{ Histology $(n=662)$} \\
\hline & \multicolumn{4}{|c|}{ Benign lesions $(n=500)$} & \multicolumn{3}{|c|}{ Carcinomas $(n=162)$} \\
\hline & $\begin{array}{l}N G \\
(n=404)\end{array}$ & $\begin{array}{l}F A \\
(n=86)\end{array}$ & $\begin{array}{l}H A \\
(n=4)\end{array}$ & $\begin{array}{l}T \\
(n=6)\end{array}$ & $\begin{array}{l}F C \\
(n=20)\end{array}$ & $\begin{array}{l}P C \\
(n=133)\end{array}$ & $\begin{array}{l}M C \\
(n=9)\end{array}$ \\
\hline Benign $(n=356)$ & 295 & 37 & 1 & 6 & 4 & 12 & 1 \\
\hline Malignant $(n=114)$ & 4 & 5 & 0 & 0 & 2 & 98 & 5 \\
\hline Indeterminate $(n=148)$ & 72 & 39 & 3 & 0 & 12 & 20 & 2 \\
\hline Unsatisfactory $(n=44)$ & 33 & 5 & 0 & 0 & 2 & 3 & 1 \\
\hline
\end{tabular}

NG, nodular goiter; FA, follicular adenoma; HA, hürthle cell adenoma; T, thyroiditis; FC, follicular carcinoma; PC, papillary carcinoma; MC, medullary carcinoma.

Table 2 Correlation of frozen section diagnoses with definitive histological diagnoses

\begin{tabular}{|c|c|c|c|c|c|c|c|}
\hline \multirow[b]{3}{*}{ Frozen section $(n=586)$} & \multicolumn{7}{|c|}{ Histology $(n=586)$} \\
\hline & \multicolumn{4}{|c|}{ Benign lesion $(n=433)$} & \multicolumn{3}{|c|}{ Carcinoma $(n=153)$} \\
\hline & $\begin{array}{l}N G \\
(n=342)\end{array}$ & $\begin{array}{l}F A \\
(n=82)\end{array}$ & $\begin{array}{l}H A \\
(n=3)\end{array}$ & $\begin{array}{l}T \\
(n=6)\end{array}$ & $\begin{array}{l}F C \\
(n=16)\end{array}$ & $\begin{array}{l}P C \\
(n=129)\end{array}$ & $\begin{array}{l}M C \\
(n=8)\end{array}$ \\
\hline Benign $(n=445)$ & 339 & 77 & 3 & 6 & 3 & 16 & 1 \\
\hline Malignant $(n=134)$ & 2 & 2 & 0 & 0 & 11 & 113 & 6 \\
\hline Deferred $(n=7)$ & 1 & 3 & 0 & 0 & 2 & 0 & 1 \\
\hline
\end{tabular}

NG, nodular goiter; FA, follicular adenoma; HA, hürthle cell adenoma; T, thyroiditis; FC, follicular carcinoma; PC, papillary carcinoma; MC, medullary carcinoma.

diagnosis underwent thyroid surgery in cases of a rapid growing nodule, local compression symptoms or for cosmetic reasons. In 586 of the 662 patients, the resected specimens were submitted for frozen sections. The tissue slices were cut in a cryostat $\left(-21^{\circ} \mathrm{C}\right)$ at $6 \mu \mathrm{m}$ and stained with haematoxylin and eosin. The frozen section diagnoses were made by experienced pathologists and classified as benign, malignant or deferred. Multiple sections from blocks of suspicious lesions were examined to make the final histological diagnosis. The diagnostic correlations of FNA cytology, frozen section, and both FNA cytology and frozen section with definitive histological diagnoses were evaluated. A microcarcinoma was defined as a tumour $\leqslant 1 \mathrm{~cm}$ in diameter. Mean tumour sizes were expressed as mean (SD). Statistical analysis was performed using $\chi^{2}$ test. Statistical tests were considered significant at $\mathrm{p}<0.05$.

\section{Results}

Correlation of cytological diagnosis by FNA cytology with the final histological diagnosis is shown in table 1. Among 662 patients, histological examination revealed benign lesions in 500 patients (404 with nodular goiters, 86 with follicular adenomas, 6 with thyroiditis, and 4 with hürthle cell adenomas) and malignancy in 162 patients (133 with papillary carcinomas, 20 with follicular carcinomas, and 9 with medullary carcinomas). FNA cytology indicated a benign diagnosis in 356 cases, which was incorrect in 17 of these cases $(4.8 \%)$. FNA cytology indicated a malignant diagnosis in 114 cases, but the final histological assessment was benign in 9 of these cases (7.9\%). In 148 cases, an indeterminate diagnosis was made and the definitive histological diagnosis was malignant in 34 of these cases $(23 \%)$. FNA cytology was unsatisfactory for diagnosis in 44 patients and 38 of these were diagnosed as benign by the final histological study. FNA cytology identified malignancies in 105 patients preoperatively $(64.8 \%$ of thyroid carcinomas).

Intraoperative frozen section determination was performed in 586 of 662 patients. Corresponding frozen section and final histological diagnoses are shown in table 2 . Frozen section diagnosis was benign in 445 cases, which was incorrect in 20 of these cases $(4.5 \%)$. Frozen section studies showed malignancies in 134 patients, but the final histological diagnosis was benign in 4 of these cases (3\%). Frozen section identified 130 of 153 thyroid carcinomas (85\%).

The correlation of FNA cytology/frozen section with final histological diagnosis is shown in table 3 . When FNA cytology and frozen section diagnoses were concordant (B/B and $M / M)$, diagnostic accuracy reached $98 \%$. The mean (SD) diameter of the eight carcinomas incorrectly diagnosed by both FNA cytology and frozen section was $1.8(1.2) \mathrm{cm}$. Records of tumour size were available in 90 of the 96 patients whose carcinoma was identified concordantly by both techniques. The mean tumour diameter in this group was $2.9(2.4) \mathrm{cm}$. The frequency of microcarcinomas was $50 \%$ (4 of 8 ) in the group with incorrectly diagnosed carcinomas and $6.7 \%$ (6 of 90 ) in the correctly diagnosed group $(p=0.0011)$. No false positive results were observed when FNA cytology and frozen section were concomitantly positive for malignancy. When FNA cytology and frozen section diagnoses were discordant, the diagnostic accuracy of frozen sections was significantly better than that of FNA cytology $(78.9 \% v 21.1 \%, p=0.0012)$. The diagnostic accuracy of frozen sections achieved $92.6 \%$ in the group of patients with an inconclusive FNA cytology diagnosis and a diagnostic frozen section biopsy (In/B, In/M, Un/B and $\mathrm{Un} / \mathrm{M})$. Of the seven cases inconclusive by both FNA cytology and frozen section, three 
Table 3 Correlation of fine needle aspiration and frozen section diagnoses with final histological diagnoses

\begin{tabular}{|c|c|c|c|c|c|c|c|c|c|c|}
\hline \multirow[b]{2}{*}{$\begin{array}{l}\text { Final histological } \\
\text { diagnoses }\end{array}$} & \multicolumn{10}{|c|}{ Fine needle aspiration diagnoses/Frozen section diagnoses } \\
\hline & $\begin{array}{l}B / B \\
(n=302)\end{array}$ & $\begin{array}{l}B / M \\
(n=7)\end{array}$ & $\begin{array}{l}M / B \\
(n=12)\end{array}$ & $\begin{array}{l}M / M \\
(n=96)\end{array}$ & $\begin{array}{l}\operatorname{In} / B \\
(n=109)\end{array}$ & $\begin{array}{l}\operatorname{In} / M \\
(n=28)\end{array}$ & $\begin{array}{l}\text { In/Def } \\
(n=6)\end{array}$ & $\begin{array}{l}U n / B \\
(n=22)\end{array}$ & $\begin{array}{l}U n / M \\
(n=3)\end{array}$ & $\begin{array}{l}\text { Un/def } \\
(n=1)\end{array}$ \\
\hline Benign $(n=433)$ & $\begin{array}{r}294 \\
8\end{array}$ & 1 & 9 & 0 & $\begin{array}{r}101 \\
8\end{array}$ & 3 & 3 & 21 & 0 & 1 \\
\hline
\end{tabular}

B, benign; $M$, malignant; In, indeterminate; Un, unsatisfactory; Def, deferred.

proved to be malignant, including two follicular carcinomas and one medullary carcinoma.

\section{Discussion}

Clinical assessment for thyroid malignancy by means of physical examination, thyroid scan, and ultrasonography is not completely reliable. ${ }^{910}$ FNA cytology is the most cost effective, safe, and accurate test in the preoperative evaluation of thyroid nodules. ${ }^{910} 14$ In our series, 662 patients underwent surgical treatment after FNA cytology, and in the same period 6018 patients received FNA cytology for nodular thyroid disease. Consequently operations were avoided in most patients after the evaluation by FNA cytology. FNA cytology can be used to separate cases of thyroid nodules into those that can be followed clinically, due to low probability of malignancy, from those requiring immediate surgical intervention. Adequate specimen is necessary for the interpretation of FNA cytology. An unsatisfactory outcome was obtained in $6.6 \%$ of cases in this series, and ranged between $3 \%$ and $32 \%$ in other reports. ${ }^{481114}$ In our hospital, FNA of the thyroid and the interpretation of the cytology were both performed and interpreted by staff endocrinologists. If the smear was unsatisfactory, aspirations could be repeated immediately, therefore, reducing the number of unsatisfactory smears. High incidence of unsatisfactory aspirates usually occurs when the cytopathologists who make the diagnoses do not perform the FNA and thus cannot judge the specimen adequacy at the time of the procedure. ${ }^{11}$ In addition, development and maintenance of the skills of FNA are important influences on the unsatisfactory rate. ${ }^{5}$

In the present study the positive predictive value for malignancy by FNA cytology was $92.1 \%$, compared to other studies where it ranged from $37.7-100 \% .^{481114}$ The nine false positive results included four cytological diagnoses of papillary carcinomas; the other five were interpreted as malignant because of nuclear pleomorphism and prominent nucleoli. The positive predictive value depends on the technique and experience of the physicians and how the data are interpreted. Our positive predictive value reached $92.1 \%$ when cytological diagnosis of definitive malignancy was considered to be "true positive". If indeterminate diagnoses were grouped into the true positive category, the accuracy rate decreased to $53 \%$, as only $23 \%$ of the indeterminate diagnoses proved to be malignant in the final histological study. The negative predictive value of FNA cytology was $95.2 \%$, comparable to other reports $(69.2-97.1 \%) .^{481114}$
Many surgeons use intraoperative frozen sections to confirm the diagnosis by FNA cytology. ${ }^{3468}$ In our series, frozen sections showed a positive predictive value of $97 \%$ and a negative predictive value of $95.5 \%$. An accuracy rate of $98 \%$ was observed when frozen section and FNA cytology diagnoses were concordant. The diagnosis of thyroid carcinoma was virtually established when FNA cytology and frozen sections were concomitantly positive for malignancy, but concordant benign FNA cytology and frozen section diagnoses were associated with $2.78 \%$ false negative results. The false negatives in this group may be partially due to the small size of the tumours, four of the eight being occult carcinomas. Surgeons may be faced with a difficult decision when FNA cytology and frozen section diagnoses are discordant. In the presence of conflicting results, frozen section diagnosis is more reliable, with an accuracy rate of $79.2 \%$ compared with $21.9 \%$ for FNA cytology. We found FNA cytology to be falsely positive for malignancy in nine cases and frozen section correctly identified all nine of these lesions as benign. In the discordant group, the false positives by FNA cytology were nine of 12 patients ( $75 \%)$. Reliance on FNA cytology to determine the extent of surgery would lead to a significant number of unnecessary extended surgical operations in the discordant pairs. It would be preferable to perform limited surgery when FNA cytology shows malignancy but frozen section diagnosis is benign. Another diagnostic discrepancy occurs when a benign FNA cytology meets a frozen section diagnosis of carcinoma. In this discordant situation, only one case was falsely positive for malignancy by frozen section, whereas frozen section identified malignancies in six patients with a benign cytological diagnosis. It is thus preferable to perform a curative operation following a malignant frozen section diagnosis in the face of a benign FNA cytology diagnosis. Although in our series frozen sections were reliable in determining the extent of surgery, 16 of 129 papillary carcinomas were underdiagnosed as benign. The underdiagnosis was mainly due to the small size of the tumours. Only one papillary microcarcinoma was present on frozen sections, but all were found on final paraffin wax sections. In addition, a few follicular variants of papillary carcinomas were interpreted as follicular adenomas on frozen sections, possibly because of the loss of nuclear morphological details caused by freezing the tissue and the tumour encapsulation resembling follicular adenomas. ${ }^{16}$

FNA cytology definitively identified only $64.8 \%$ of thyroid carcinomas preoperatively 
whereas frozen section identified $85 \%$ of malignancies. This discrepancy is mainly due to the number of indeterminate or suspicious diagnoses by FNA cytology. Indeterminate cytological diagnosis-that is, follicular neoplasm, accounted for $22.4 \%$ of cases in our series. The diagnosis of follicular carcinoma is defined by evidence of vascular or capsular invasion and it is impossible to evaluate these criteria by FNA cytology. ${ }^{17}{ }^{18}$ It is sometimes impossible to distinguish benign from malignant follicular neoplasm even using frozen sections because of the limited number of tissue blocks that can be investigated intraoperatively. We identified 11 of 16 follicular carcinomas by frozen section (68.8\%) and six of the 11 were minimally invasive carcinomas. FNA cytology was not helpful in the diagnosis of follicular carcinoma. The inability to differentiate follicular carcinomas from adenomas and hyperplastic thyroid nodules by FNA cytology resulted in a high incidence of indeterminate lesions in several series. ${ }^{37811}$ The frequency of malignancy in indeterminate FNA cytology in our series was $23 \%$, comparable to the $13-36 \%$ in other reports. ${ }^{37811}$ However, $63 \%$ of our benign cases with an indeterminate cytological diagnosis were hyperplastic nodules within a nodular goiter, this seems to be a relatively high number compared with $30-69 \%$ in other series. $^{37811}$ In areas of endemic goiter like Taiwan, there is a high incidence of multiple adenomas, which are histologically difficult to differentiate from hyperplastic nodules. ${ }^{19}$ In this situation, our pathologists tend to interpret the results as hyperplastic nodules rather than adenomas, this possibly explains why almost two thirds of histologically benign cases with indeterminate FNA cytology were shown to be hyperplastic nodules. Twenty cases of papillary carcinoma were cytologically interpreted as indeterminate. This is because some follicular variants of papillary carcinomas (lack of papillary fronds, intranuclear cytoplasmic inclusions or nuclear grooves on cytology smears) were categorised as indeterminate. In addition, a few papillary carcinomas were found to be occult and the aspirations were performed on a follicular adenoma or hyperplastic nodule. Excluding seven patients with a deferred frozen section diagnosis, the accuracy of frozen section examination in our patients with indeterminate FNA cytology was $92 \%$. There were only three false positives and eight false negatives in 137 indeterminate cytological diagnoses. Because of its high accuracy in this series, frozen section proved to be a valuable procedure for intraoperative decision making in patients with an indeterminate cytological diagnosis.

Multinodular and large goiters are common in Taiwan, therefore FNA cytology alone may not give a definitive diagnosis. Our surgeons frequently performed additional histological examinations by frozen section to help the decision making during the surgery. By using frozen section as the final guide for the extent of thyroidectomy, limited operations such as lobectomy and isthmectomy are usually performed in patients with thyroid nodules. If the frozen section diagnosis is benign, the operation is terminated. In Chang Gung Memorial Hospital, total thyroidectomy is performed for papillary, follicular, and medullary thyroid carcinomas $^{20}$; therefore, if a frozen section identifies a malignancy, extended surgery is performed. The surgeons were given consent by the patients to define the extent of thyroid resection according to the diagnosis of frozen section. In our series, 23 patients (3.9\% of 586) underwent a second operation because of a false negative or deferred frozen section diagnosis. However, only four patients $(0.68 \%)$ received excessive thyroid surgery based on the impression given by frozen section. Our conclusions about combining FNA cytology and frozen section results in intraoperative decision making are similar to the recommendations of some authors. ${ }^{3468}$ The relative reliability of FNA cytology and frozen section varies across environments, giving rise to discrepancies in establishing principles for intraoperative decision making. Rodriguez et al found that intraoperative biopsy by frozen section was useful only in thyroid nodules with a suspicious cytology but aided little in a malignant or benign cytological diagnosis. Keller $e t ~ a l^{12}$ and Hamburger and Hamburger ${ }^{13}$ suggested that FNA cytology alone provided sufficient information for determining the extent of thyroid resection in a benign or malignant cytological diagnosis. In a suspicious FNA cytology result, frozen sections did not have sufficient sensitivity for determining the extent of resection.

In conclusion, FNA cytology is an accurate preoperative test for the evaluation of thyroid nodular disease and helps to distinguish malignant from benign lesions for the purpose of selecting lesions that need surgical intervention. Intraoperative frozen section is a valuable procedure for confirming the cytological diagnosis and especially important in identifying malignancies in cases with an indeterminate or unsatisfactory cytological diagnosis. With reliance on frozen sections as an intraoperative guide of thyroid surgery, unnecessary extensive surgery and second operations can be considerably reduced.

1 Hsiao YL, Chang TC. Prevalence of goiter in Taiwanese adults: a preliminary study. $\mathcal{f}$ Formos Med Assoc 1995;94:197-9.

2 Mazzaferri EL. Thyroid cancer in thyroid nodule: finding a needle in the haystack. Am $\mathcal{f}$ Med 1992;93:359-62.

3 Layfield LJ, Mohrmann RL, Kopald KH, Giuliano AE. Use of aspiration cytology and frozen section examination for of aspiration cytology and frozen section examination for Cancer 1991;68:130-4

4 Schmid KW, Ladurner D, Zechmann W, Feichtinger H. Clinicopathologic management of tumors of the thyroid gland in an endemic goiter area. Combined use of preoperative fine needle aspiration biopsy and intraoperative frozen section. Acta Cytol 1989;33:27-30

5 Hall TL, Layfield LJ, Philippe A, Rosenthal DL. Sources of diagnostic error in fine needle aspiration of the thyroid. Cancer 1989;63:718-25.

6 Shaha AR, Dimaio T, Webber C, Jaffe BM. Intraoperative decision making during thyroid surgery based on the results of preoperative needle biopsy and frozen section. Surgery 1990;108:964-71.

7 Rodriguez JM, Parrilla P, Sola J, Bas A, Aguilar J, Moreno A, et al. Comparison between preoperative cytology and intraoperative frozen section biopsy in the diagnosis of thyroid nodules. Br ₹ Surg 1994;81:1151-4.

8 Bugis SP, Young JE, Archibald SD, Chen VS. Diagnostic accuracy of fine-needle aspiration biopsy versus frozen secaccuracy of fine-needle aspiration biopsy versus frozen sec-
tion in solitary thyroid nodules. Am $尹$ Surg 1986;152:41116. 
9 Griffies WS, Donegan ED, Abel ME. The role of fine-needle aspiration in the management of the thyroid nodule. Laryngoscope 1985;95:1103-6.

10 Aggarwal SK, Jayaram G, Kakar A, Goel GD, Prakash R Pant CS. Fine needle aspiration cytologic diagnosis of the solitary cold thyroid nodule. Comparison with ultrasonography, radionuclide, perfusion study and xeroradiography. Acta Cytol 1989;33:41-7.

11 Altavilla G, Pascale M, Nenci I. Fine needle aspiration cytology of thyroid gland diseases. Acta Cytol 1990;34:251-

12 Keller MP, Crabbe MM, Norwood SH. Accuracy and significance of fine-needle aspiration and frozen section in determining the extent of thyroid resection. Surgery 1987;101:632-5.

13 Hamburger JI, Hamburger SW. Declining role of frozen section in surgical planning for thyroid nodules. Surgery 1985 98:307-12.

14 Godinho-Matos L, Kocjan G, Kurtz A. Contribution of fine needle aspiration cytology to diagnosis and management of thyroid disease. 7 Clin Pathol 1992;45:391-5.
$15 \mathrm{Riu} \mathrm{CH}$. On the studies of the methods of staining blood film (Romanowsky's system). Fournal of the Niigata Medical Association 1956;70:635-43

16 Tielens ET, Sherman SI, Hruban RH, Ladenson PW. Follicular variant of papillary thyroid carcinoma. A clinicopathologic study. Cancer 1994;73:424-31.

17 Löwhagen T, Sprenger E. Cytologic presentation of thyroid tumors in aspiration biopsy smear. A review of 60 cases. Acta Cytol 1974;18:192-7.

18 Miller JM, Kini SR, Hamburger JI. The diagnosis of malignant follicular neoplasms of the thyroid by needle biopsy. Cancer 1985;55:2812-7.

19 Droese M. Cytological aspiration biopsy of the thyroid gland. New York: Schattauer Press, 1980.

20 Lin JD, Weng HF, Huang MJ, Huang BY, Huang HS, Jeng LB. Thyroid cancer treated in Chang Gung Memorial Hospital (northern Taiwan) during the period 1979-1992: clinical presentation, pathological finding, analysis of prognostic variables, and results of treatment. $\mathcal{f}$ Surg Oncol 1994;57:252-60. 